\title{
ARQUITECTOS DE LA ACADEMIA ESPAÑOLA EN ROMA (SIGLO XIX) *
}

\author{
POR \\ ESTEBAN CASADO ALCALDE \\ Escuela Técnica Superior de Arquitectura \\ Universidad Politécnica de Madrid
}

\begin{abstract}
Siendo el presente texto la segunda y última entrega del asunto a que se refiere el título, cabe simplemente especificar que se trata de un seguimiento de ciertos comportamientos académicos -los más relacionados con la actividad de unos pensionados en Italia, tales como los envíos de pensión o los viajes por el extranjero, y sus consecuencias en su trayectoria posterior, cuales son las dedicaciones docentes y las huellas de todo ello perceptibles en su producción- que unos determinados artistas, los arquitectos, manifiestan como consecuencia de su paso por esa institución española en el exterior que es nuestra Academia en Roma.

Palabras clave: Academia española en Roma. Arquitectura española. Siglo XIX.

This text is the second and last part of a study on architects in the Spanish Academy of Rome. Academic practices are examined -principally related to artists on scholarship in Italy- such as the designation of pensions and foreign travel, and their subsequent repercussions in the trajectory of these architects, whether in their architectural productions or as professors. It is clear that all those who passed through this Spanish institution abroad were affected by the experience.
\end{abstract}

Key words: Spanish Academy in Rome. Spanish architecture. $19^{\text {th }}$ century

\section{Segunda promoción (1878-1881)}

Para la concesión de las dos pensiones de número de la $2^{\mathrm{a}}$ Promoción hay unas primeras oposiciones en 1878, obteniendo plaza por unanimidad el único candidato, Manuel ZaBaLA GALLARDo. En una segunda convocatoria de ese mismo 1878 obtiene la otra pensión por mayoría Juan MonTSERRAT Vergés, a quien, de acuerdo con el reglamento vigente de 1877, que estipulaba como primer ejercicio (que era teórico) escribir sobre algún punto importante de teoría e historia del arte, le corresponderá exponer el tema del sistema de construcción de la Arquitectura romana; para el segundo ejercicio, consistente en copiar del yeso un fragmento de Arquitectura, le corresponde en suerte (a partir de un sorteo entre tres de épocas griega, romana y renacentista) el fragmento renacentista.

* El texto de este artículo es la segunda parte de la ponencia del mismo título cuya primera mitad fue ya publicada en las Actas de las «XI JoRnAdAs DE ARTE» (El Arte español fuera de España) celebradas en Madrid en noviembre de 2002 de acuerdo con la convocatoria del Departamento de Historia del Arte del Instituto de Historia del C.S.I.C. (Madrid, 2003, pp. 63-77). 
En cuanto a la prueba definitiva, proyectar un monumento arquitectónico, ésta se subdividía en dos nuevos ejercicios: en el primero, a realizar en 12 horas seguidas, se presentaba el proyecto dibujado sin escala pero bien determinado en plantas y alzados: sería a nuestro juicio la continuidad de aquella prueba «de repente» de los viejos Premios Generales de Arquitectura convocados por la Academia de San Fernando; el segundo y último ejercicio, el desarrollo del proyecto, se ejecutaba en tres meses pero entonces con la posibilidad de consulta de las obras, estampas o fotografías oportunas: se trataría ahora del equivalente de la prueba «de pensado» en seis meses de los antiguos concursos académicos, bien que la realización de ambas pruebas sea en orden inverso, puesto que en las oposiciones a Roma se trata de dos ejercicios de la misma prueba, en tanto que en la corporación fernandina eran dos pruebas totalmente distintas. La continuidad del fenómeno radica, a nuestro juicio, en el carácter de organismo «técnico» que San Fernando asume para el Ministerio, que puede aprovechar así su larga experiencia en enviar pensionados a Roma. De nuevo en este caso que nos ocupa, sorteados los temas redactados por el Tribunal entre los cuales se encontraban «Museo Arqueológico», «Templo de glorias nacionales», Circo ecuestre», «Estación principal de camino de hierro», «Universidad»y «Escuela de Arquitectura», es este último el que le toca ejecutar a Monserrat ${ }^{1}$.

El desarrollo de la pensión en el caso de Zabala consiste en la entrega, para el primer año, de la reproducción del basamento de la columna de Trajano, según lo estipulado reglamentariamente sobre copiar un notable detalle o fragmento de Arquitectura antigua a gran tamaño y con el correspondiente estudio de perfiles, puesto que no hay modificación respecto del reglamento anterior de octubre de 1873 en este punto de las obligaciones de los pensionados. Obtendrá la calificación «honrosa» u honorífica. Su segundo envío se produce tras fijar su residencia en Venecia, donde proyecta el estudio de la iglesia bizantina de Torcello, que —según el informe trimestral de 1-VII-1879 de Casado del Alisal- es uno de los tipos más interesantes y originales del arte oriental-véneto de los siglos viII al x. Será su estado de salud, con fiebres provocadas por lo insalubre del clima, el que motive su cambio por San Marcos de Venecia en esa obligada copia del estado actual de un monumento perteneciente a las buenas épocas del arte, trabajo que le proporcionará calificación «satisfactoria». En cuanto al tercer envío, donde sí cambia lo pedido respecto a la reglamentación anterior, puesto que se sustituye el proyecto de restauración de un monumento de las buenas épocas del arte por el «proyecto de un edificio de utilidad pública», elige el de una casa o palacio municipal de distrito, siendo calificado como «inferior» a lo exigido por el Reglamento ${ }^{2}$.

Pese a este decrescendo en calificaciones, Loredo, que sitúa a Zabala como el único que va a Roma de entre un grupo de arquitectos titulados en Madrid entre 1872 y 1876, tales Adaro, Magdalena, Lázaro, Fort y Landecho, subraya de los que denomina sus excelentes envíos, ése de San Marcos de Venecia, añadiendo que a su vuelta a España se dedicó por completo a la enseñanza en la Escuela madrileña, desempeñando la clase de Dibujo de conjunto con ejemplar celo. Completa la breve semblanza el haber restaurado en Toledo el Cristo de la Luz, la dirección en ese momento en que escribe Loredo (1925) de la restauración de San Juan de los Reyes (continuando así la gran tarea de Mélida), y la elección como secretario de la Academia de San Fernando a la muerte de Repullés ${ }^{3}$.

\footnotetext{
${ }^{1}$ Margarita Bru Romo, La Academia Española de Bellas Artes en Roma (1873-1914), Madrid, 1971, pp. 63-64 y 363 (filiación de Montserrat, nacido en Reus, Tarragona, en 1853, hijo de Juan [confitero] y Ana). El pensionado Montserrat consigue la propuesta el 10-V-1879 y el nombramiento el 16-V del mismo; la toma de posesión es el 15-IX siempre del mismo 1879, y el cese por renuncia en noviembre de 1880 (Esteban Casado Alcalde, La Academia Española en Roma y los pintores de la primera promoción, Madrid, Universidad Complutense (col. «Tesis Doctorales»), tomo I, pp 261-262, 263).

${ }^{2}$ Bru, ob. cit., pp. 148 y 219.

${ }^{3}$ Román Loredo, «La Arquitectura», en «Apéndice. Arte español desde principios del siglo xIx hasta el mundo actual», t. VI (Arte Moderno y Contemporáneo, 1750-1924) de la Historia del Arte en todos los tiempos y pueblos, de Karl Woermann, Madrid, 1925, pp. 636 y 638.
}

$A E A$, LXXVII, 2004, 306, pp. 129 a 138 
En efecto, en la vertiente de profesor y académico, destacamos de Zabala un ejemplar manuscrito de un proyecto docente para la asignatura de «Teoría general del Arte Arquitectónico» en la Escuela madrileña, fechado en junio de 1896, que aquí no podemos sintetizar pero del que ya anticipamos que de su programa no se aleja mucho (aunque esté más desarrollado, en dos cursos) el que confeccionará 34 años más tarde otro ex pensionado, Albiñana Chicote, como impreso de 1930. Asimismo, y también manuscrito pero de 1921, Zabala da un «Informe sobre la reforma del plan de estudios de la Escuela de Arquitectura de Madrid», en el que tampoco podemos detenernos.

En cuanto académico, está el testimonio de su discurso de ingreso en la corporación fernandina en 1917 sustituyendo a Fernández Casanova, de quien hace el protocolario elogio y entre cuyos datos biográficos señala el haber sido «discípulo muy distinguido del maestro $D$. Miguel Aguado», bien que haya sido también advertido el directo discipulado de Casanova respecto a Juan de Madrazo. Ahora bien, el asunto del discurso de Zabala versa acerca «Del barroquismo en la Arquitectura», estableciendo precedentes para sus reflexiones en trabajos de crítica e historia de la Arquitectura barroca nacional de Cabello Aso, Lampérez y Danvila, así como en discursos e informes académicos de Arturo Mélida, Velázquez Bosco, Repullés y Landecho. Por eso señala ya como punto de partida el hecho de que la Academia de San Fernando tenga asumida ya esa valoración estética. Basándose en un recorrido histórico, sus consideraciones le llevan a advertir en la aplicación del ornato barroco una actitud ecléctica, sin trascendencia ni finalidad fuera de sí mismo.

Más allá de la contestación de Landecho a Zabala incluyendo los datos biográficos del recipiendario y profundizando en las reflexiones sobre la arquitectura barroca, tendríamos aquí el testimonio de un reconocimiento ya generalizado del neobarroco como una tendencia nacionalista más, la última en ese momento tras la plateresca; y es que, potenciada por la revalorización del papel de Madrid en el siglo XVIII - lo que en efecto reforzaría más su faceta casticista - y justificada a partir, entre otros, del discurso de ingreso académico de Mélida en 1899 defendiendo la arquitectura barroca, esa tendencia neobarroca, plasmada en fachadas con carácter movido y artístico, triunfaría entre 1900 y 1920, tal como se ha sabido precisar ${ }^{4}$.

La pensión de Juan Montserrat se reduce a un único envío, un fragmento del arco de Tito que obtiene calificación satisfactoria, porque tras viajar en su segundo año para elegir nuevo trabajo, decide renunciar en noviembre de ese mismo 1880. Tampoco sabemos prácticamente nada de su trayectoria posterior: previamente había sido nombrado arquitecto de la diócesis de Guadix-Baza y del arzobispado de Granada por real orden de 15 de diciembre de 1877, es decir, inmediatamente antes de la pensión. Probablemente la necesidad de incorporarse a un cargo que exigía residir en la circunscripción donde hubiese de prestar sus servicios o, de otra forma, la imposibilidad de guardar el puesto para cuando regresara de Roma, es lo que le haría renunciar a la pensión. En efecto, sabemos que la tardanza de Montserrat en asumir su tarea creó intranquilidad en la Junta Diocesana por la acumulación de expedientes sin resolver, y que a instancias del prelado y a través del Ministerio de Gracia y Justicia, Montserrat comunicaba la aceptación del nombramiento, se disculpaba por hallarse todavía en Madrid y prometía una inmediata toma de posesión, que finalmente se produjo el 20 de marzo de 1878, en una rara ocasión en que pisó la diócesis.

Señalado el dato de que el arquitecto diocesano no tenía sueldo fijo, se entiende la preca-

\footnotetext{
${ }^{4}$ Loredo, «La Arquitectura», ob. cit., p. 660. Pedro Navascués Palacio, Arquitectura y arquitectos madrileños del siglo XIX, Madrid, 1973, pp. 258 y 317-318. Para la labor de Zabala en la restauración arquitectónica, Isabel Ordieres Díaz, Historia de la Restauración Monumental en España (1835-1936), Madrid, 1995, pp. 452 a 458 (en Addenda IV de los Apéndices), recogiendo su autoría en diversos informes de distintos monumentos dentro de los trabajos de la Comisión Central de Monumentos entre 1907 y 1933.
} 
riedad del cargo en términos generales; en cuanto al caso particular de Montserrat, evidenciada su reiterada incomparecencia, se decide a dimitir por real orden de 7 de mayo de 1888 «fundado en sus muchas ocupaciones como arquitecto municipal y diocesano de Granada». Sin embargo, al año siguiente, en 1889 , renuncia igualmente al cargo de arquitecto de la propia diócesis de Granada ${ }^{5}$.

\section{Quinta promoción (1889-1893)}

El salto a esta quinta promoción se produce porque ni la tercera (1881/82-1885) ni la cuarta (1884/85-1888/89) llevan arquitectos ${ }^{6}$. Y anticipamos que tampoco los habrá en la sexta promoción (1895-1899) ni en la séptima (1900-1904) que ya se introduce en el siglo xx. Solamente a partir de 1904 (octava promoción, 1904-1908) parece solucionado el problema al advertirse un normalizado flujo de los mismos a Roma ${ }^{7}$. Ya examinamos en su momento bajo un epígrafe de «absentismo de los arquitectos» ${ }^{8}$, ése su desinterés por beneficiarse del mecenazgo estatal en las dos últimas décadas del siglo xIx. Si recordamos ahora el dato es porque entre las propuestas avanzadas por el Ministerio para la ocupación de las plazas de Roma, está la del nombramiento automático de los que han sido pensionados como profesores supernumerarios de la Escuela Superior de Arquitectura o de otras Escuelas como las de Bellas Artes; también, el que tuvieran preferencia para los nombramientos de arquitectos de la Administración o en las contratas de obras públicas y medidas similares ${ }^{9}$.

Pero ya en la $5 .^{\text {a }}$ promoción y vigente todavía el reglamento de 1877 , la convocatoria de octubre de 1888 para la plaza de mérito es respondida por tres candidatos: el ya conocido ex pensionado de número Amador de los Ríos (quien, aunque con méritos suficientes, queda fue-

5 José Manuel Rodríguez Domingo, «La Junta de Reparación de Templos de la diócesis de Guadix-Baza (1845-1904)», en Cuadernos de Arte de la Universidad de Granada, t. 31 (2000), pp. 159-175, concretamente pp. 173-174. I. Ordieres, Historia de la Restauración Monumental..., pp. 356-357 (Addenda II, Arquitectos y Juntas diocesanas, Archidiócesis de Granada y Diócesis de Guadix, donde se dice que este arquitecto [Montserrat] en 1887 todavía no había ido a reconocer ninguna iglesia y en 1888, ante la petición de explicaciones, dimite). Allí mismo (p. 44) referencia a la figura del arquitecto diocesano y a facultativos de otra dedicación, así como testimonio documental (p. 232, doc. $\mathrm{n}^{\circ}$ 6) de la problemática en que se veía inmersa la Junta diocesana de Granada en ese mismo momento (concretamente en 1891).

${ }^{6}$ La plaza de mérito y una de las de número en la tercera promoción se convocan en febrero de 1882 . Vacante ya de antes la otra por la renuncia de Montserrat, ha de ser la adjudicada a Muñoz Degrain en diciembre de 1881 aunque como pensionado de mérito en pintura, ya que al no haberse presentado ninguna solicitud para la pensión de número de arquitectura, anunciada en agosto de ese mismo 1881 pero ya en segunda convocatoria, el Ministerio se había reservado el derecho a disponer de dicha vacante en la forma que estimara conveniente. En cuanto a las convocatorias de febrero de 1882 (para las vacantes de Rabanal, de mérito, y Zabala, de número) ese desinterés de los arquitectos por las plazas de Roma que ya venía manifestándose desde unos años antes, obliga a un segundo llamamiento en abril del mismo 1882, tras los dos meses reglamentarios de presentación de solicitudes. La ausencia de candidatos igualmente para esta segunda ocasión será lo que acabe por preocupar al Ministerio de Estado y le impulse a solicitar de la Academia de San Fernando la información pertinente para analizar y remediar el absentismo de los arquitectos. En lo que respecta a la cuarta promoción, ni siquiera se convocan las plazas de arquitectura, puesto que el Ministerio está en ese momento estudiando nuevas fórmulas para hacer más atractivas a los arquitectos las pensiones de Roma. Por ello, un extenso informe trimestral del director Palmaroli de fecha 10-I-1886, al dar el estado en ese momento de las pensiones vacantes o próximas a vacar, señala entre las primeras las tres de arquitectura: la de mérito vacante desde noviembre del 81 (Rabanal), una de número desde noviembre del 81 también (Zabala), y la otra de número desde noviembre del 80 (Montserrat). (Véase E. Casado Alcalde, La Academia Española en Roma..., I, pp. 263-270. También Bru [ob. cit.] se hace eco de este absentismo de los arquitectos: «Durante todos estos años todas las plazas de la sección de Arquitectura estuvieron sin cubrir» [p. 155]).

${ }^{7}$ Para las promociones del siglo xx, y más concretamente las del primer tercio, véase E. Casado Alcalde, «La Academia de Roma entre 1900 y 1936», en Catálogo Exposición «Roma: Mito, Modernidad y Vanguardia», Roma, 1998, pp. 39-69. Existen además sucesivas entregas de este mismo autor para los otros dos tercios del siglo xx, en posteriores y consecutivos catálogos del programa de exposiciones de los pensionados, celebradas en 1999 y 2001 respectivamente.

${ }^{8}$ E. Casado Alcalde, La Academia Española en Roma..., I, pp. 233-236.

${ }^{9}$ También recoge la información Bru (ob. cit., pp. 65-66) aunque fundiendo los datos de los distintos documentos del proceso en un único episodio narrativo.

AEA, LXXVII, 2004, 306, pp. 129 a 138 


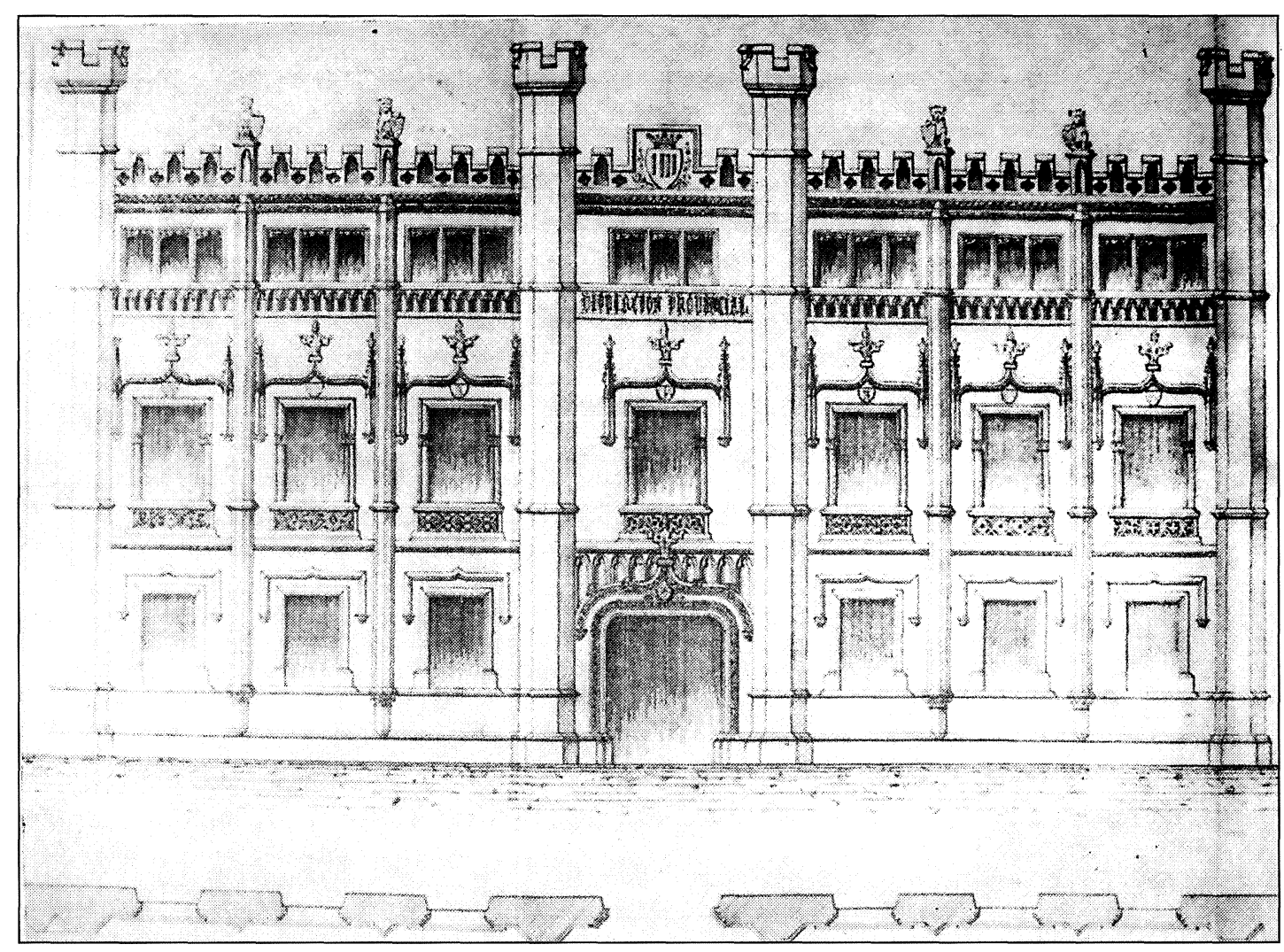

Fig. 1. Pavía: Diputación Provincial de Palma de Mallorca (fachada).

ra de concurso porque ya tiene 43 años y excede por tanto el límite de 40), Julio Martínez Zapata (que se retira para opositar a una de las pensiones de número, ejercicio que también abandona en 4-II-1889), y Joaquín Pavía Bermingham, que obtiene la plaza al quedar como único candidato ${ }^{10}$.

Durante su pensión y además de tener que actuar como testigo en el acto de pérdida de la beca por parte del escultor de mérito Querol, ordenada por Palmaroli, vemos a Pavía en su primer año ocupado con estudios de restauración de monumentos notables, entre ellos el que será su trabajo reglamentario, el Templo de las Vestales en el Foro Romano. Pero diversas tareas que le encomienda el embajador cerca de la Santa Sede, marqués de Pidal, sobre la tasación de propiedades inmobiliarias españolas en Roma paralizan sus obligaciones de pensionado, por lo que, concedida prórroga de tres meses, ha de recomenzar en 1892 el primer envío. Éste, según el informe de Palmaroli, se justifica porque hasta 1875 se desconocía el emplazamiento de ese templo de Vesta, creyéndose que era el llamado de S. Stefano Rotondo. Las excavaciones de 1871-1884 descubrieron las ruinas del templo y casa de las vírgenes vestales al pie del Palatino, y como hasta ahora no se había intentado una restauración arquitectónica, será ésa la tarea que constituya su envío, consistente en una serie de gráficos que van minuciosamente descritos por Palmaroli.

\footnotetext{
${ }^{10}$ Bru, ob. cit., pp. 82-83, 169-171 y 223. Las fechas de nombramiento y toma de posesión son respectivamente 9-IV1889 y 15 -VII del mismo (E. Casado Alcalde, La Academia Española èn Roma..., I, pp. 274 y 277). En cuanto a Julio Martínez Zapata, resultó ser un asiduo concurrente a certámenes oficiales, y sobre todo a las Exposiciones Nacionales de Bellas Artes, donde consiguió terceras medallas en 1890 y 1892 y segundas en 1897 y 1899 según el conocido libro de Pantorba (Historia y crítica de las Exposiciones Nacionales de Bellas Artes celebradas en España, $1^{\mathrm{a}}$ ed. de 1948, pp. 55
} 


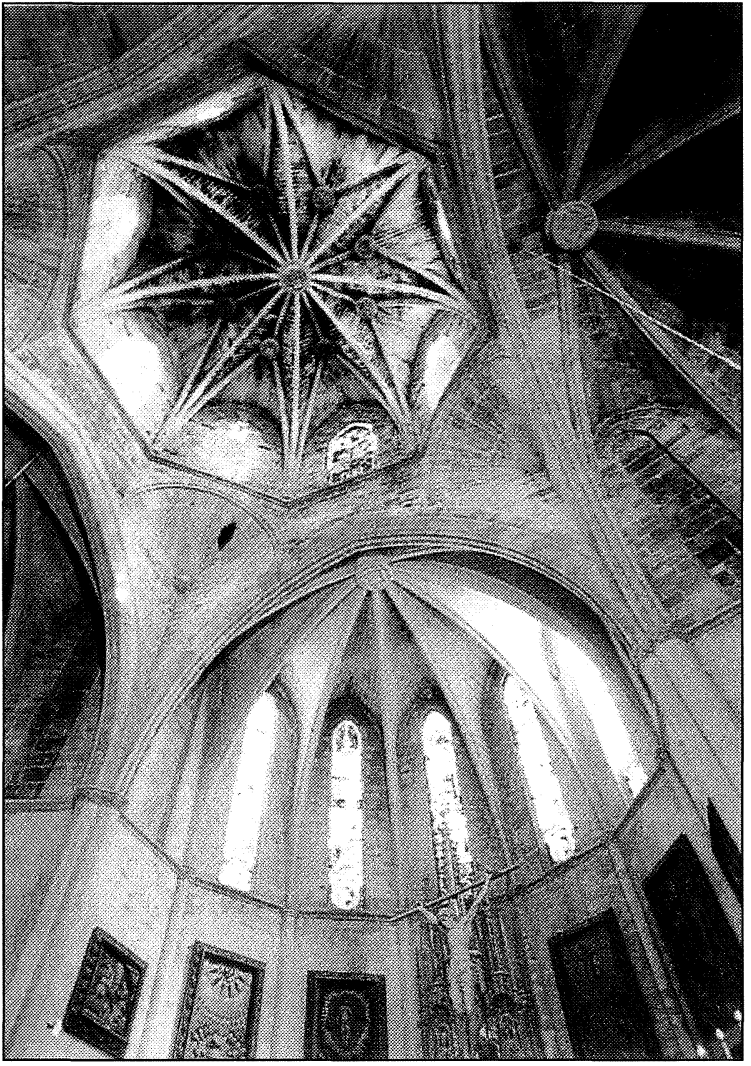

Fig. 2. Pavía: Cabecera neogótica de la iglesia de Sineu (Mallorca).

Ahora bien, se nos ocurre, al enumerar todos estos proyectos de restauración de templos y monumentos, que la elección de los mismos por parte de los pensionados pudiera ser un reflejo del avance de las excavaciones arqueológicas $\mathrm{y}$, en suma, del conocimiento progresivo de esa arquitectura antigua. De cualquier forma, $\mathrm{Pal}-$ maroli añade el elogio de que es un trabajo el de Pavía «hecho todo con sumo cuidado, mostrando relevantes cualidades como artista y profundo conocimiento de la historia de la arquitectura». Y en efecto, obtendrá la calificación honorífica en 1893.

Este proyecto de restauración fue cedido - al igual que dos de los envíos del otro arquitecto de la promoción, Albiñanapor el Ministerio a la Escuela de Arquitectura de Madrid, pero antes conoció el éxito (una medalla de primera clase) en nuestra Exposición Internacional de Bellas Artes de 1892, por lo que también se llevó a la Universal de Chicago del año siguiente, a la que Pavía irá como Comisario General de Bellas Artes de la Delegación Española y donde dejará obra. Pero antes el catálogo del certamen español —en el que

figura entre los vocales de los dos Jurados, el de Admisión y el de Colocación y Calificación, Aguado de la Sierra- además de describir los siete bastidores del proyecto nos entera de que el pensionado ha nacido en la Fábrica Nacional de Armas de Orbaiceta en Navarra (al parecer por destino de su padre, puesto que la familia era de San Sebastián) y ha estudiado en la Escuela de Arquitectura de Madrid.

La intención de Pavía para su segundo año de residir en Pau y ejecutar como último trabajo el proyecto de un edificio público en Manila, se ve truncada por el citado nombramiento de comisario español en la exposición de Chicago el 16-III-1893, lo que motiva el descontento del nuevo director de la Academia en Roma, Alejo Vera, que cree que esas distintas ocupaciones le distraerán de sus obligaciones de pensionado. Y desde luego, aunque se había compro-

y 351). Pero por la consulta de los correspondientes catálogos de dichas Exposiciones llegamos a sospechar que Martínez Zapata fue también amigo de competir en los distintos concursos abiertos para la erección de monumentos conmemorativos y la edificación de los abundantes edificios administrativos del momento: Diputación Provincial de Vizcaya según su proyecto presentado a la Exposición (en este caso Internacional) de 1892, monumento al rey D. Pelayo según el catálogo de la de 1895, Ayuntamiento de Santander según su proyecto presentado a la «General» de 1897, y particularmente un puente sobre el río Urumea en San Sebastián, que en el catálogo de la de 1904 se denomina «proyecto en ejecución» y que en efecto es el enfático y grandilocuente Puente de María Cristina, obra suya en colaboración con el ingeniero Ribera que pasaría a reflejar el papel de estos arquitectos incluyendo motivos modernistas que posibilitan hoy en la ciudad «un interesante recorrido por un modernismo de raíz francesa y, muy excepcionalmente, de matiz vienés (Pedro Navascués Palacio, Arquitectura española (1808-1914), vol. XXXIV** de la col. «Summa Artis», Madrid, 1993, p. 579). Y todavía Martínez Zapata y Rodríguez - pero anteriormente y no para ubicación determinada, invitándonos por tanto a desechar aquí la hipótesis de concurso público-, presenta otros dos proyectos de edificios oficiales y administrativos, el de una Escuela de Música para la Nacional de 1890, y el de un Palacio para Congreso y Senado, para la «General» de 1899, siempre según los correspondientes catálogos.

$A E A$, LXXVII, 2004, 306, pp. 129 a 138 
metido a realizar en Chicago su último envío, en 1894 todavía permanecía ausente. Ahora bien, lo que en Chicago hace en verdad Pavía - reflejando con ello esa maleabilidad tan propia del eclecticismo, puesto que, como a continuación veremos, también había trabajado en neogótico- es un revival neoárabe, una reproducción de las arcadas de la mezquita cordobesa (la que ni antes ni después vuelve a copiarse) en el Edificio de Manufacturas de dicha exposición ${ }^{11}$.

Pero por otro lado, al ir a Roma cualificado como pensionado de mérito, Pavía ya tenía una trayectoria anterior: profesor auxiliar de la Escuela de Arquitectura madrileña, donde explicó la mecánica aplicada, desempeñó también los cargos de arquitecto provincial de Baleares y diocesano de Mallorca desde 1877 hasta su marcha de la isla en 1884. Como facultativo en la primera de ambas dedicaciones es continuador de la obra de la Casa de Misericordia, pero sobre todo es autor del proyecto de la Diputación, cuya fachada neogótica; comenzada en 1882, presenta una evidente deuda con la Lonja de Palma. Pero también de arquitectura civil es el Circo Balear, proyectado en 1876 y calificado dentro de una corriente ecléctica cuyas aportaciones exóticas eran vistas en su época como chinescas. Como arquitecto diocesano es Director facultativo de la restauración de la Catedral, pero también autor de proyectos tanto de nueva planta, la iglesia de S'Indioteria, como de reforma, tales la reconstrucción de la iglesia parroquial de Alcudia (1882) o la cabecera neogótica costeada por el conde Joseph d'Espanya i Rossinyol de la parroquial de Sineu, en este caso siguiendo la solución de cimborrios burgaleses del siglo xv (con bóveda estrellada sobre capillas de brazos ochavados) pero con rasgos de un gótico catalán del tipo de Pedralbes por la sobriedad de huella cisterciense.

Tras la pensión y de regreso a San Sebastián, le vemos desarrollar también una carrera de conferenciante y acumular cargos y nombramientos: correspondiente de las Academias de San Fernando y de la Historia en Guipúzcoa, Miembro de la Comisión de Monumentos de la misma provincia en 1904, e incluso diputado provincial (1896-1905), entre los de mayor proyección nacional, al lado de condecoraciones y encomiendas ${ }^{12}$.

En cuanto a la convocatoria de las plazas de número de esta 5. ${ }^{a}$ promoción, las oposiciones duran de enero a mayo de 1889. Julio Martínez Zapata se retira antes de comenzar el primer ejercicio y queda como único opositor, consiguiendo finalmente la pensión, Alberto ALBIÑANA CHICOTE, con lo que vuelve a quedar una plaza vacante ${ }^{13}$.

De cualquier forma, es de apreciar ese cierto éxito temporal que parece haber obtenido el Ministerio con sus medidas para motivar a los arquitectos, pues en esta ocasión se cubren al

\footnotetext{
${ }^{11} \mathrm{M}^{\mathrm{a}}$ José Bueo Fidel, Arquitectura y nacionalismo. (Pabellones españoles en las Exposiciones Universales del siglo XIX), Málaga, 1987, p. 54 (con fotografía). Javier Hernando, Arquitectura en España, 1770-1900, Madrid, 1989, p. 246. José Manuel Rodríguez Domingo, La arquitectura «neoárabe» en España: el medievalismo islámico en la cultura arquitectónica española (1840-1930), Tesis Doctoral, Granada, 1997, II, pp. 248 y 263. P. Navascués, Arquitectura y arquitectos madrileños..., p. 269, y Arquitectura española..., p. 331.

${ }^{12}$ Espasa (Enciclopedia Universal Ilustrada Europeo Americana), vol. 42, 1920 (reed. 1991), p. 1006. Gran Enciclopedia de Mallorca, vol. 13, Inca, Mallorca, 1989, p. 23 (voz de Belén Roger García). C. Cantarellas Camps, La arquitectura mallorquina desde la Ilustración a la Restauración, Palma de Mallorca, 1981, pp. 321, 478, 513-514 y 515. P. Navascués, Arquitectura española..., p. 454 (dando el dato de la titulación de Pavía en 1887). I. Ordieres, Historia de la Restauración Monumental..., pp. 360 y 384. El propio Pavía es autor de publicaciones como Las Catacumbas de Roma. Conferencias pronunciadas por ... Seguidas de una noticia acerca de la vida y obras del arqueólogo romano Juan Bautista de Rossi, Madrid, 1895 (con datos en la «Noticia» sobre el descubrimiento conmemorativo de un bajorrelieve del también pensionado Marinas, en un acto académico de 1892, con importante intervención institucional española entre la que se cita a nuestra Academia en Roma); y también de autoría de Pavía es el artículo «La imagen de San Ignacio del Seminario de Vergara», en Euskal-Erría, t. XLIX (1903), pp. 85-90.

${ }^{13} \mathrm{Bru}, o b$. cit., pp. 74 y 83 . La vacante de arquitectura recae en el pintor pensionado de número Enrique Simonet. En cuanto a los límites cronológicos de la pensión de Albiñana, la propuesta es de 29-V-1889, el nombramiento de 13-VI, la toma de posesión el 15-VII siempre de 1889, y el cese, tras la concesión de un año de prórroga, el 1-VIII-1893 (E. Casado Alcalde, La Academia Española en Roma..., I, pp. 274-275 y 277). Añadamos, por último, que este Alberto Albiñana Chicote es calificado como «arquitecto y escultor» en ese Diccionario de la Arquitectura española (p. 21) de José Luis Morales y Marín que forma el tomo 6 de la Historia de la Arquitectura española (Zaragoza, 1987) dirigida por el mismo autor.
}

AEA, LXXVII, 2004, 306, pp. 129 a 138 
menos dos de sus tres plazas reglamentarias, aunque también podría darse como elemento de influencia un dato que se ha apuntado -bien que como factor negativo- sobre las fuentes de inspiración más corrientes de los arquitectos en formación durante los últimos años del siglo XIX y primeros del XX: dentro de las obras de consulta habituales de los estudiantes de la Escuela de Madrid estaban algunas publicaciones francesas, y entre ellas las ediciones de los «Premios de Roma» de la École des Beaux Arts de París. Ahora bien, en esta línea de dar cada vez más peso a la información bibliográfica en el desarrollo de los artistas, no sobra aquí el citar un catálogo de la biblioteca de la Academia que se remite desde Roma al Ministerio en 1893, donde sobresalen respecto a la arquitectura Llaguno, Caveda, Ceán, Lomazzo, Rada y Delgado, Demetrio de los Ríos, Viollet-le-Duc o Vasari ${ }^{14}$.

En cuanto a las actividades reglamentarias de ese último pensionado del siglo XIX que es Albiñana, cabe decir que se emplea el primer año en el análisis del arco de Tito, que se le califica como de haber cumplido «con exceso» sus obligaciones (pues había ampliado el estudio del fragmento a todo el arco), y que depositado el envío en la Escuela madrileña, la acuarela del conjunto pudo verse en la exposición antológica de la Academia en $1979^{15}$. Para los trabajos de segundo año viaja en 1891 por Grecia y el Alto Egipto, empleándose especialmente en el estudio del estado en ese momento del templo de Horus en Karnak y la restauración de un fragmento. Pero si hasta aquí se igualaba con el pensionado del primer momento Amador de los Ríos en cuanto al estudio de lo egipcio, Albiñana consigue visitar Constantinopla también, para luego viajar por ciudades europeas y asentarse en París para el tercer envío. Entre tanto se le concede en diciembre de ese mismo 1891 la máxima calificación por el segundo.

Suponemos que es éste, aunque lleve el nombre de «Templo de Rous en Tebas (Egipto)» ${ }^{16}$, el que junto al primer envío, «Estudios del Arco de Tito en Roma», presenta años después a la Exposición en ese momento llamada General de Bellas Artes de 1897, obteniendo sólo medalla de segunda clase por el templo egipcio, aunque en descargo de Albiñana esté el que quedara desierta la adjudicación de la medalla de primera clase. El pensionado madrileño se habría presentado al certamen tratando de superar la tercera medalla obtenida en la Exposición de 1892 con un «Proyecto de una puerta de Madrid», que parece ser la «Puerta monumental» que constituye su tercer envío del que hablaremos a continuación.

Acabada su estancia física en la Academia - lo que motiva elogios por su comportamiento y la valoración de «artista inteligente y estudioso»- el tercer año lo ocupa primeramente con un proyecto de teatro que cambiará después por una «Puerta monumental», según comunica el nuevo director Alejo Vera. En la calificación de ese tercer envío, en 1893, aunque se tiene en cuenta que se trata de un proyecto de monumento que sólo se pide a los pensionados de mérito, se advierte que la memoria reglamentaria no resultaba buena. De cualquier forma y pese a ello, consigue prórroga de una año con la que recorre ciudades castellanas como Ávila, Segovia, Alcalá, Toledo y otras, en busca de asunto para nueva entrega, que será, ya en 1893, un proyecto de Ministerio de Estado, el cual, al ser producto de la prórroga y no el tercer envío que quedaba de propiedad del pensionado, decide ceder al Gobierno.

\footnotetext{
${ }^{14}$ El dato de los libros franceses en la Escuela de Madrid, en Carlos Flores, Arquitectura española contemporánea. I (1880-1950), Madrid, 1989 (reed. de la de 1961), p. 93, nota con asterisco [*]. Lo referente a la biblioteca de la Academia Española en Roma, en E. Casado Alcalde, La Academia Española en Roma..., I, p. 240.

${ }^{15}$ Reproduce el arco de Tito a escala de 0,05 p.m. y va firmada «Alberto Albiñana, Roma, 1890», dando fe por nuestra parte de su presencia en la citada exposición pese a no llegar a registrarse en el catálogo. Los datos de la pensión en Bru, ob. cit., pp. 171-172 y 223, incluyendo -como en todos los casos anteriores y que aquí no hemos querido reproducir para no recargar más el texto- los tribunales calificadores.

${ }^{16} \mathrm{Si}$ no se trata de una errata (por Horus) sería una variante escrita y tal vez fonética de Rahes, también escrito Iahes, «entidad mal conocida, que se identifica con Horus-Min en el capítulo 17 del Libro de los Muertos» (Isabelle Franco, Pequeño diccionario de mitología egipcia, Palma de Mallorca-Barcelona, 1994, pp. 65 y 107).
}

$A E A$, LXXVII, 2004, 306, pp. 129 a 138 

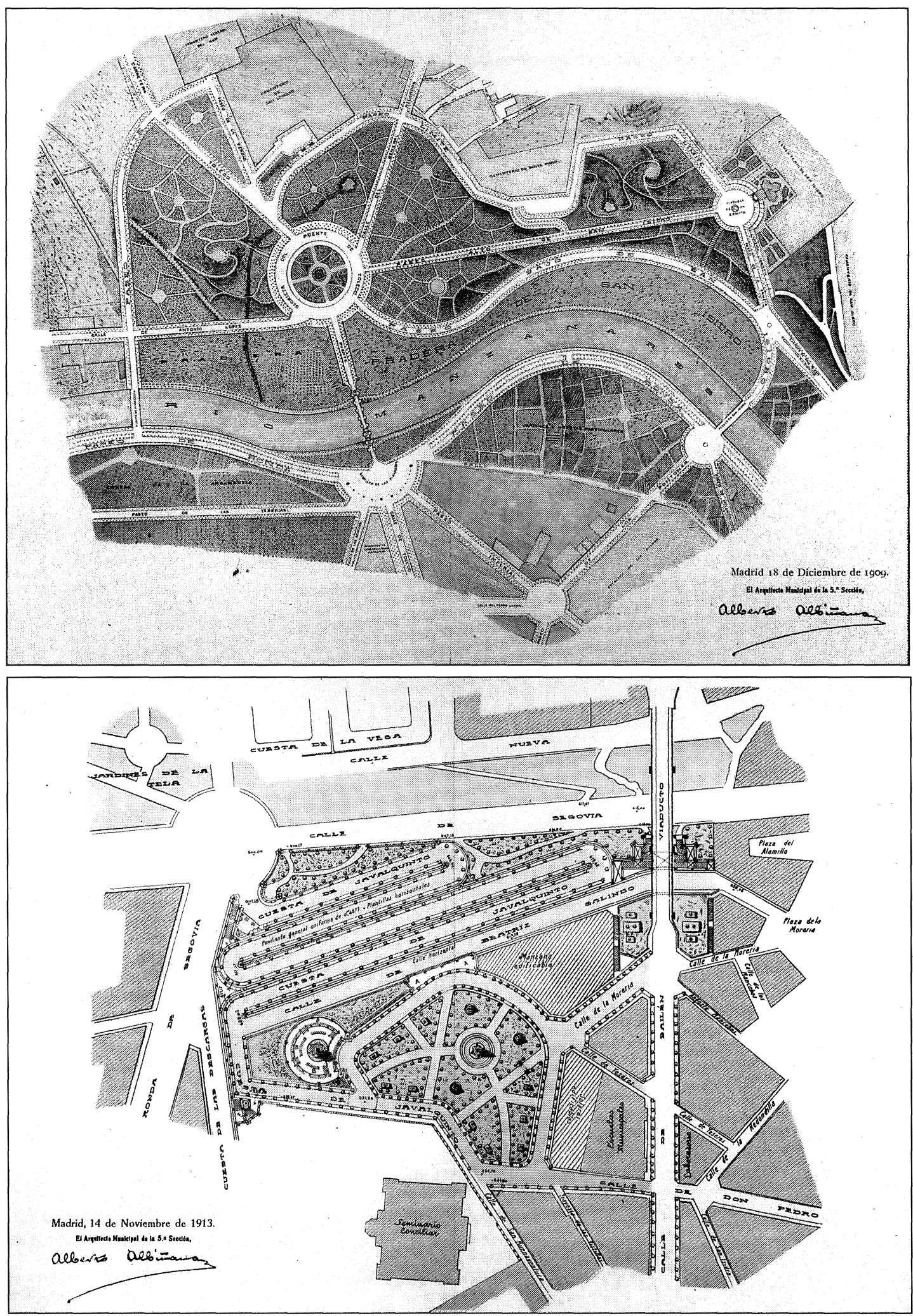

Fig. 3. Albiñana Chicote: Proyecto de aislamiento del Puente de Toledo en Madrid.

Fig. 4. Albiñana Chicote: Proyecto de reforma de Las Vistillas en Madrid. 
Poco podemos añadir, finalmente, sobre la trayectoria posterior de Albiñana. Si ya de estudiante en la Escuela madrileña confeccionó unos manuscritos «Apuntes de construcción: curso de 1885 a 1886 / tomados en clase por A. Albiñana y Chicote», de docente en la misma publica el Programa de dos cursos de Teoría del Arte Arquitectónico (Madrid, 1930) como profesor numerario de la expresada enseñanza, programa que ya hemos advertido sigue de cerca - aunque con la lógica mayor profundización en los temas- al que Zabala incluía en su manuscrito proyecto docente de «Teoría general del arte arquitectónico» de 1896.

En cuanto a su actividad profesional y como arquitecto municipal de la Quinta Sección, le conocemos dos proyectos de remodelación urbana de dos significativos puntos madrileños: el primero, Proyecto de aislamiento del Puente de Toledo, formación de una glorieta a la entrada del mismo y parque comprensivo de la pradera de San Isidro y de la Ermita del Santo Patrón de Madrid, con su memoria y planos así como la moción de aprobación por parte del alcalde Luis Silvela, aunque publicado en Madrid en 1918, en realidad estaba confeccionado nueve años antes, el 18 de diciembre de 1909, y se trataba de desembarazar el puente de casas y huertas adyacentes, añadir puentes auxiliares por la imposibilidad de ensanchar el histórico y trazar vías y una plaza en el extrarradio, al otro extremo del puente. El segundo es el Proyecto de reforma de alineaciones y urbanización del Campillo de las Vistillas, también con informe y planos, y publicado dos años después (Madrid, 1920) pero igualmente confeccionado con anterioridad, el 14 de noviembre de 1913; en este caso, de menor envergadura, se trata de convertir en plaza regularizada dentro de esa especial topografía del terreno el erial entonces existente y - además de las consiguientes alineaciones- modificar el trazado excesivamente pendiente de la cuesta de Javalquinto como objetivo secundario.

Y concluimos con un dato anecdótico de la biografía de Albiñana: su viaje a Burdeos para hacerse cargo el 5 de junio de 1899 del cuerpo de Goya y trasladarlo a España, contagiado ciertamente el arquitecto de una tacañería respecto a las honras fúnebres ${ }^{17}$ que tal vez procediera del Gobierno; el encargo oficial le vino de su amigo el marqués de Pidal, embajador cerca de la Santa Sede hasta 1893, o sea, durante la pensión de Albiñana, y en ese momento recién nombrado ministro de Fomento del gobierno formado por Francisco Silvela en marzo de 1899.

\footnotetext{
${ }^{17}$ Nieves Concostrina y Jesús Nuño, «Goya: un traslado sin pies "ni cabeza"», en Adiós, Revista de empresas, III, n 11 , septiembre-octubre de 1998.
}

$A E A$, LXXVII, 2004, 306, pp. 129 a 138 